\title{
Welches ist die Standardtherapie bei älteren Menschen?
}

Fragestellung: Ist die Kombination aus Strahlentherapie und Chemotherapie mit Temozolomid den jeweiligen Monotherapien bei älteren Patienten mit Glioblastom überlegen?

Hintergrund: Eine Temozolomid-Chemotherapie begleitend zur Strahlentherapie und in sechs Erhaltungszyklen danach ist die Standardtherapie des Glioblastoms bei Patienten bis 70 Jahre in gutem Allgemeinzustand. Die Standards der Therapie bei der wachsenden Population älterer Menschen mit Glioblastom bleiben umstritten: Nach den Leitlinien der European Association for Neurooncology (EANO) sollte bei diesen Patienten der O6-Methylguanin-DNA-Methyltransferase (MGMT)-Promotor-Methylierungsstatus bestimmt werden, um die Patienten dann bei Methylierung einer alleinigen Chemotherapie und bei fehlender Methylierung einer alleinigen Strahlentherapie zuzuführen.

Rusthoven CG, Koshy M, Sher DJ et al. Combined-modality therapy with radiation and chemotherapy for elderly patients with glioblastoma in the temozolomide era. A national cancer database analysis. JAMA Neurol 2016; 73: 821-8
Dieser molekularstratifizierten Therapie steht die Hypothese entgegen, dass alle Patienten unabhängig vom Alter, sofern aufgrund von Fitness und Komorbidität vertretbar, kombiniert mit einer Strahlen- und einer Chemotherapie behandelt werden sollten.
Patienten und Methodik: Bei der hier vorgestellten Studie handelt es sich um eine retrospektive Kohortenstudie auf der Basis von Daten der National Cancer Data Base. Es wurden prospektiv Daten von neu diagnostizierten Glioblastompatienten im Alter von 65 Jahren oder älter einbezogen, die zwischen Januar 2005 und Dezember 2011 die Diagnose erhalten hatten. Analysiert wurde der Zusammenhang von Therapie und Überleben unter Berücksichtigung einiger wichtiger prognostischer Faktoren.

Ergebnisse: Insgesamt wurden 16.717 Patienten in die Untersuchungskohorte eingeschlossen. Das mediane Überleben betrug neun Monate mit kombinierter Behandlung, 4,7 Monate mit Strahlentherapie allein, 4,3 Monate mit Chemotherapie allein und 2,8 Monate, wenn keine weitere spezifische Therapie erfolgte.

Die kombinierte Behandlung blieb auch in der multivariaten Analyse den beiden Monotherapien überlegen, während sich kein Unterschied zwischen Chemotherapie allein und Strahlentherapie allein zeigte.

Schlussfolgerungen: Nach Ansicht der Autoren legt ihre Analyse nahe, dass die kombinierte Behandlung der Einzeltherapie überlegen ist. Daraus ziehen sie die Schlussfolgerung, dass geeigneten älteren Patienten eine kombinierte Radio-Chemotherapie angeboten werden sollte.

\section{Warten auf die randomisierten Daten}

Die Stärke der Studie ist die große Anzahl der Patienten, zu den Schwächen zählen das Fehlen molekularer Marker, insbesondere von MGMT, sowie fehlende Informationen zur Rezidivtherapie. Die Schlussfolgerung ist moderat gehalten: Schon jetzt werden ältere Patienten, die biologisch deutlich jünger wirken, wie jüngere Patienten kombiniert mit Radio-Chemotherapie behandelt, zumindest in den meisten Zentren.

Auch wenn die Analyse inhaltlich gut gemacht ist, bleibt es dennoch eine Illusion, dass man durch Modellieren und multivariate Analysen randomisierte Studien überflüssig machen kann. In der Tat wurde zumindest ein Teil der zugrunde liegenden Frage in einer kürzlich auf dem ASCO-Kongress vorgestellten Studie eines kanadisch-europäischen Konsortiums beantwortet. In dieser Studie zeigte sich in Abhängigkeit vom MGMT-Status tatsächlich eine Überlegenheit der kombinierten Therapie gegenüber alleiniger Strahlentherapie, aber eben erneut in der Patientenpopulation, die primär als geeignet für eine kombinierte Behandlung empfunden wurde. Somit bleibt der Eindruck, dass die Studie einmal mehr eine „self-fulfilling prophecy" bestätigt, nämlich dass Patienten mit günstigen prognostischen Faktoren aggressiver behandelt werden und länger überleben. Der Kausalzusammenhang zwischen aggressiverer Therapie und längerem Überleben bleibt angesichts der Patientenselektion aber offen.

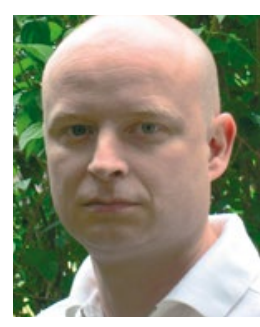

Prof. Dr. med. Michael Weller, Zürich/Schweiz

Direktor der Klinik für Neurologie, Universitätsspital Zürich E-Mail:michael.weller@usz.ch 